OPEN ACCESS

Edited by:

Fabio Galbusera,

University of Ulm, Germany

Reviewed by:

Bruce P. Ayati,

University of lowa, USA

Andy L. Olivares,

Pompeu Fabra University, Spain

*Correspondence:

Ivan Argatov

ivan.argatov@gmail.com

Specialty section:

This article was submitted to Biomechanics, a section of the journal Frontiers in Bioengineering and

Biotechnology

Received: 13 September 2016 Accepted: 11 October 2016 Published: 01 November 2016

Citation:

Argatov I and Mishuris G (2016) Articular Contact Mechanics from an Asymptotic Modeling Perspective:

A Review.

Front. Bioeng. Biotechnol. 4:83. doi: 10.3389/fbioe.2016.00083

\section{Articular Contact Mechanics from an Asymptotic Modeling Perspective: A Review}

\author{
Ivan Argatov ${ }^{1 *}$ and Gennady Mishuris ${ }^{2}$ \\ ${ }^{1}$ Institut für Mechanik, Technische Universität Berlin, Berlin, Germany, ${ }^{2}$ Institute of Mathematics and Physics, Aberystwyth \\ University, Ceredigion, UK
}

In the present paper, we review the current state-of-the-art in asymptotic modeling of articular contact. Particular attention has been given to the knee joint contact mechanics with a special emphasis on implications drawn from the asymptotic models, including average characteristics for articular cartilage layer. By listing a number of complicating effects such as transverse anisotropy, non-homogeneity, variable thickness, nonlinear deformations, shear loading, and bone deformation, which may be accounted for by asymptotic modeling, some unsolved problems and directions for future research are also discussed.

Keywords: articular contact, knee joint, articular cartilage, asymptotic model, thin layer, biphasic theory, deformation, damage

\section{INTRODUCTION}

Articular cartilage is a non-vascular soft tissue, which covers the ends of bones and thereby prevents damage in their contact. In the knee joint, a half of body weight is transferred through the articular contact in a quiet standing position, and the level of loading increases progressively in walking, running, and jumping. Experimental investigations (van den Bogert et al., 1999) have shown that joint contact forces of up to $300 \%$ body weight can occur even during normal walking, and may rise to $550 \%$ during the push-off phase of running, whereas various skiing activities produce a joint contact force ranging from $400 \%$ body weight (long turns and flat slope) to $900 \%$ body weight during short turns on a steep slope.

The mechanical aspects of articular contact, such as the contact pressure pattern [e.g., which is changed due to some gait disorders (Rosneck et al., 2007)], the maximum level of loading, or the type of loading [e.g., impact (Herzog and Federico, 2006; Kessler et al., 2006; Garcia et al., 2008)] are closely related to he development and progression of osteoarthritis (Maly et al., 2008). On the other hand, the analytical modeling of articular contact is necessary in formulating equations for the reaction forces generated in joints during multibody simulations of human and animal movements (Delp and Loan, 2000; Machado et al., 2011).

With a tremendous progress in development of computer simulation tools, the early analytical models of articular contact (Eberhardt et al., 1990; Blankevoort et al., 1991; Eberhard et al., 1999; Bei and Fregly, 2004) were succeeded by FE models (Wu et al., 1997; Caruntu and Hefzy, 2004; Wilson et al., 2005a; Galbusera et al., 2014) that have been steadily improved in accuracy and realistic presentation of the contacting parts (Caruntu and Hefzy, 2004) as well as their complexity has been increased by accounting for many factors such as microstructure (Bursać et al., 2000), meniscus (Peña et al., 2006), fluid exudation (Carter et al., 2004), which are usually neglected in analytical studies. 
Asymptotic modeling is a mathematical modeling approach aimed to simplify a given mathematical model by considering the so-called limit situation with respect to a certain dimensionless parameter. As a result of asymptotic modeling, we obtain an asymptotic model, which bears the main features of the original mathematical model essential for the chosen limit situation (Argatov, 2012a).

\section{ASYMPTOTIC MODELING OF ARTICULAR CONTACT}

\subsection{Asymptotic Model for Deformation of Articular Cartilage}

It is well known (Poole et al., 2001) that articular cartilage mainly consists of extracellular matrix and interstitial fluid. Namely, this biphasic nature of cartilage was reflected in the mathematical model for its deformation response developed by Mow et al. (1980). In order to describe the deformation response of articular cartilage layer under an external load, a number of modeling issues should be fixed (including, the geometry of cartilage layer, the method of its loading, and the boundary conditions imposed at the layer surfaces).

Figure 1A shows a biphasic layer bonded to an impermeable rigid base and loaded by an axisymmetric distributed normal load, which, for the sake of simplicity, does not change in time. Evidently, the problem contains a dimensionless parameter of geometrical nature $\varepsilon=h / a$, where $h$ is the layer thickness and $a$ is the radius of the loaded area. Under the assumption that $\varepsilon \ll 1$, it becomes possible to look for the solution in the limit situation as $\varepsilon \rightarrow 0$ in the form of a series with respect to the parameter $\varepsilon$ by applying the corresponding perturbation technique. In this way, an asymptotic solution of the axisymmetric deformation problem for an isotropic biphasic layer was obtained by Ateshian et al. (1994).

It should be emphasized that articular cartilage can be regarded as a time-dependent material. So that its response to a suddenly applied normal pressure exhibits two limit situations in time: namely, the short-time response and the long-time (equilibrium) response, and it is interesting to observe (Ateshian et al., 1994; Barry and Holmes, 2001) that the instantaneous response of a biphasic tissue corresponds to that of an incompressible elastic material, whereas in the long-time regime (in the equilibrium state), the biphasic layer responds as a compressible material.

The axisymmetric asymptotic model by Ateshian et al. (1994) and Wu et al. (1996) was generalized for non-axisymmetric loading configurations (Argatov and Mishuris, 2011a) and extended to the cases of a transversely isotropic biphasic/viscoelastic layer (Argatov and Mishuris, 2011c, 2015b) and of a thin biphasic poroviscoelastic layer (Argatov and Mishuris, 2015c).

In particular, the first-order asymptotic solution for the normal displacements, $w_{0}(t, \mathbf{y})$, of the surface points of a bonded thin biphasic layer is obtained in the form

$$
w_{0}(t, \mathbf{y})=-\frac{h^{3}}{3 G^{\prime}} \Delta_{y} p(t, \mathbf{y})-h k_{1} \int_{0}^{t} \Delta_{y} p(\tau, \mathbf{y}) d \tau
$$

Here, $p(t, \mathbf{y})$ is a distributed surface load, which depends on the time variable $t$ and the Cartesian coordinates $\mathbf{y}=\left(y_{1}, y_{2}\right)$ on the layer surface, $\Delta_{y}=\partial^{2} / \partial y_{1}^{2}+\partial^{2} / \partial y_{2}^{2}$ is the Laplace differential operator, $G^{\prime}$ is the out-of-plane shear modulus of the solid matrix, $k_{1}$ is the transverse (in-plane) permeability.

\subsection{Contact Problem Formulation}

In order to formulate the articular contact problem, it is first necessary to list the key mechanical quantities that play a major role in the contact phenomena. First of all, this is the pair of the contact force, $F(t)$, and the contact approach, $\delta_{0}(t)$, both being functions of time. The contact force $F$ represents the total of external load, which is transferred through the joint, while $\delta_{0}$ represents the corresponding so-called generalized displacement and has a meaning of the normal displacement between the bones (whose deformation is usually neglected). Second, this is the pair of the contact pressure, $p$, and the layer deformations represented by the surface normal displacements, $w_{0}^{(1)}$ and $w_{0}^{(2)}$. The latter quantities can be regarded as internal variables (there is no way of non-invasive measuring the contact pressures in an intact joint).

Further, another important aspect of contact interactions between the cartilage layers is determined by the geometry of the layers, which, in turn, determines the gap function, $\varphi\left(y_{1}, y_{2}\right)$ (see Figure 1B). The case of subchondral bones shaped as elliptic paraboloids [a commonly assumed geometry in the Hertzian theory of elastic contact (Johnson, 1985)] can be regarded as the main approximation for the tibiofemoral contact in the weight-bearing region [in particular, it covers the case of spherical bones assumed by Ateshian et al. (1994) and Wu et al. (1996)] and was introduced by Koo and Andriacchi (2007).

Thus, taking into account the contact condition of nonpenetration inside the contact area, $\omega$, that is

$$
w_{0}^{(1)}(t, \mathbf{y})+w_{0}^{(2)}(t, \mathbf{y})=\delta_{0}(t)-\varphi(\mathbf{y}), \quad \mathbf{y} \in \omega(t),
$$
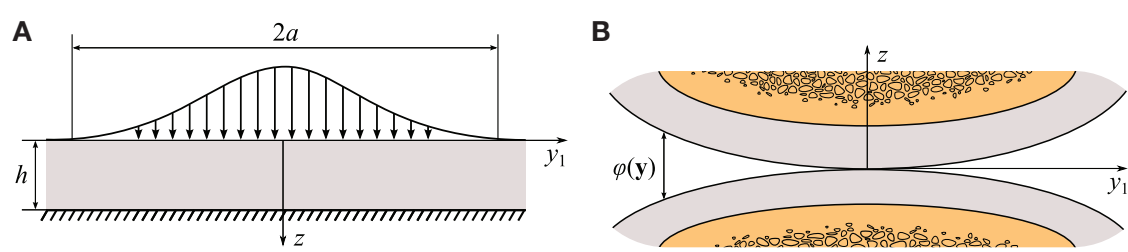

FIGURE 1 | (A) Model problem for the deformation of articular cartilage layer [after Ateshian et al. (1994)]; (B) schematics of the initial contact geometry between two cartilage layers bonded to subchondral bones. 
and equation (1), which relates $w_{0}^{(n)}(t, \mathbf{y}), n=1,2$, to the contact pressure $p(t, \mathbf{y})$, we arrive at the equation

$$
\Delta_{y} p(t, \mathbf{y})+\chi \int_{0}^{t} \Delta_{y} p(\tau, \mathbf{y}) d \tau=m\left(\varphi(\mathbf{y})-\delta_{0}(t)\right),
$$

where the coefficients $\chi$ and $m$ are given by

$$
\chi=3\left(\frac{G_{1}^{\prime} k_{1}^{(1)}}{h_{1}^{2}}+\frac{G_{2}^{\prime} k_{1}^{(2)}}{h_{2}^{2}}\right), \quad m=3\left(\frac{h_{1}^{3}}{G_{1}^{\prime}}+\frac{h_{2}^{3}}{G_{2}^{\prime}}\right)^{-1} .
$$

Of course, the approximate (asymptotic) mathematical model [equation (3)] is restricted to the short-time contact period, thereby may be oversimplifying many aspects of articular contact (some of them will be discussed later), including the assumption of direct contact between the cartilage layers without taking into account the influence of meniscus or interstitial fluid [see, e.g., the discussion given by Ateshian et al. (1994)].

\subsection{Effect of Boundary Conditions}

First of all, we emphasize that the contact area $\omega$ is not known in advance and depends on the value of the contact force

$$
F(t)=\iint_{\omega(t)} p(t, \mathbf{y}) d \mathbf{y}
$$

In order to be able to solve equation (3) uniquely, it is necessary to formulate two boundary conditions on the contour $\Gamma(t)$ of the domain $\omega(t)$. One condition is obvious and follows from the continuity of the contact pressure, which is absent outside the contact area, i.e.,

$$
p(t, \mathbf{y})=0, \quad \mathbf{y} \in \Gamma(t),
$$

Concerning another boundary condition

$$
\frac{\partial p}{\partial n}(t, \mathbf{y})=0, \quad \mathbf{y} \in \Gamma(t)
$$

where $\partial / \partial n$ is the normal derivative, in the literature, there was a discussion (Hlaváček, 1999; Wu and Herzog, 2000; Argatov et al., 2016a).

Apart from the fact that the mathematical model [equations (3), (5), and (6)] incorporates the model for instantaneous response, it was shown (Argatov and Mishuris, 2015b) that the boundary conditions [equations (5) and (6)] are asymptotically exact for thin incompressible elastic layer in unilateral contact. At the same time, the question of imposing refined boundary conditions [like those introduced by Hlaváček (1999) in the axisymmetric case] is still open and requires the study of the corresponding boundary layer problem for a 2D biphasic strip.

On the other hand, when formulating the contact problem, a refined contact condition [instead of equation (2)] can be used, as shown by Mishuris and Argatov (2009) and Argatov and Mishuris (2010) in the axisymmetric case. The refined condition takes into account the tangential displacements, which undergo the contacting points during the contact deformation, thereby increasing the complexity of the contact problem in the non-axisymmetric case (Rogosin et al., 2016) and introducing a certain correction into the solution (namely, the relation between the contact force and the contact approach turns out to be most susceptible to the effect of tangential displacements).

\subsection{Some Implications Drawn from the Asymptotic Models}

Simple as it is, the asymptotic model [equations (3)-(6)], as applied in the axisymmetric case by Wu et al. (2000), sheds light on the influence of the degenerative changes in the articular cartilage mechanical properties on the contact pressure distribution. In particular, it is known (Korhonen et al., 2002) that the articular cartilage superficial zone, which is characterized by tangentially oriented collagen fibrils, is important for the deformation response of the articular cartilage layer. The effect of superficial zone was recently modeled (Argatov and Mishuris, 2016) by an extensible membrane coating attached to the surface of a thin bonded incompressible elastic layer, and it was shown that the reinforcing effect reduces the out-of-plane shear compliance of the elastic layer up to a maximum of four times (in the limit situation of an inextensible membrane).

When the asymptotic model for deformation of a thin biphasic layer [equation (1)] was generalized for a transversely isotropic layer (Argatov and Mishuris, 2015b), it highlighted the roles played by the transverse shear modulus $G^{\prime}$ and the in-plane permeability $k_{1}$. Recall that, while the shear modulus, $G$, for an isotropic material is related to its Young's modulus, $E$, and Poisson's ratio, $v$, via the formula $G=E /[2(1+v)]$, in the case of a transversely isotopic material $G^{\prime}$ represents a material property independent from those measured in the confined and unconfined compression tests.

\subsection{Average Characteristics for Articular Cartilage Layer}

It is known that articular cartilage layers are inhomogeneous, anisotropic, non-uniform, and non-flat (Schinagl et al., 1997; Mow and Guo, 2002). At the same time, equations (1) and (3) operate with constant characteristics $h, G^{\prime}, k_{1}, R_{1}$ and $R_{2}$. The question of the model sensitivity with respect to the parameter variations was studied in a number of papers (Anderson et al., 2010; Argatov and Mishuris, 2011b; Argatov, 2013a). In particular, in the case of a thin transversely isotropic and transversely homogeneous (TITH) elastic layer, the average transverse shear modulus, $\bar{G}^{\prime}$, is given by the following formula (Argatov and Mishuris, 2015b):

$$
\bar{G}^{\prime}=\left(\frac{3}{h^{3}} \int_{0}^{h} \frac{z^{2} d z}{G^{\prime}(z)}\right)^{-1} .
$$

At the same time, the average thickness and curvature radii of the gap function depend on the extend of the contact area, over which the averaging is performed (Argatov, 2012a).

\subsection{Contact Force Modeling for Multibody Simulations}

There is a vast literature on modeling of reaction contact forces generated in joints (Silva et al., 1997; Flores et al., 2011; Machado et al., 2011; Monteiro et al., 2011). A majority of the employed models represent variations of the following model introduced by Hunt and Crossley, 1975:

$$
F=b k x^{n} \dot{x}+k x^{n} .
$$


Here, $n$ is a real constant, $k$ is a stiffness coefficient, and $b$ is a damping parameter.

A peculiarity of the force-displacement relation [equation (7)] is that the value of the force $F$ returns to zero, when the displacement value $x$ does the same. In biomechanical applications, equation (7) was used in a number of papers (Silva et al., 1997; Guess et al., 2010; Machado et al., 2010).

At the same time, the contact force model, which is based on the asymptotic model [equations (3)-(6)], shows a residual deformation, when the contact force vanishes. The same phenomenon is seen in viscoelastic models even for such simple as Maxwell and Kelvin-Voigt models (Argatov, 2013b; Argatov et al., 2016b).

However, it is known (Selyutina et al., 2015) that in the case of cyclic dynamic loading of a viscoelastic solid, the steady-state response will be analogous to that of the Hunt-Crossley model [equation (7)]. Therefore, by considering a steady-state response of the asymptotic model, we have established a link between the two models and expressed the coefficients $k$ and $b$ in equation (7) in terms of the biphasic layer parameters.

\subsection{Damage and Fracture Criteria}

It goes without saying that the damage and fracture phenomena in articular cartilage, which occur under loads exceeding the physical level (Aspden et al., 2002), are too complicated (Peña, 2011) to be captured by such simple analytical models as equations (3)-(6).

However, the simple analysis turns out to be very useful for identifying (Argatov and Mishuris, 2015a,d) the modeling framework of the laboratory impact tests (Jeffrey et al., 1995; Varga et al., 2007). Indeed, though the damage and fracture processes are multiscale, they are governed by the level of external loading during the impact event, whose evolution can be monitored at the macros-scale. In particular, by inspecting the coefficient of restitution, one can estimate the share of the impact energy dissipated or spent on the damage accumulation and surface fissuring or formation of small cracks.

\section{UNSOLVED PROBLEMS AND DIRECTIONS FOR FUTURE RESEARCH}

\subsection{Nonlinearity}

Articular cartilage is a soft tissue and may undergo (moderately) large deformations without damage (Quinn et al., 2001; Morel and Quinn, 2004). However, the asymptotic model [equations (3)-(6)] was developed in the framework of the linear biphasic theory, and thus, its extension to the case of deformations, which cannot be regarded as small, will be very useful. Here, it is worth mentioning the known dependency of the cartilage permeability on the volumetric strain (Mow et al., 1980), which also was not accounted for by the linear asymptotic models, while this effect dumps the deformation very quickly and, thereby, making the fitting of experiments by asymptotic models quite difficult. It seems, furthermore, that Soltz and Ateshian (2000) obtain excellent results both in tension and compression by adopting a conewise linear elasticity (Curnier et al., 1995).

\subsection{Compound Asymptotic Model for Merging the Short- and Long-Time Responses}

Recall that the asymptotic model [equations (3)-(6)] was developed to capture the short-time asymptotics (Ateshian et al., 1994), and it leads to unrealistic predictions as $t \rightarrow \infty$ (in particular, the contact approach is unbounded). On the other hand, the equilibrium response of the biphasic layer can be modeled by that of a compressible elastic layer. Thus, there are two asymptotic models, which could be merged into the so-called compound asymptotic model.

\subsection{Triphasic Model}

As a generalization of the biphasic theory for articular cartilage (Mow et al., 1980), the so-called triphasic theory, which combines the biphasic theory with the physico-chemical theory for ionic and polyionic solutions, has been developed by Lai et al. (1991). There is an undoubted interest in formulating the deformation problem for a thin triphasic layer and constructing its first-order asymptotic solution.

\subsection{Meniscus}

From a geometrical point of view, articular cartilage can be modeled as a layer (of variable thickness). A meniscus has a more complicated geometry (Peña et al., 2006), and, to the best of the authors' knowledge, there is a lack of a simple analytical (approximate or asymptotic) model for the deformation response of menisci. Consequently, a generalization of the asymptotic model [equations (3)-(6)], which incorporates the meniscus deformation, will be useful, since the menisci transfer a significant proportion of the load across the knee joint (Fahmy et al., 1983).

\subsection{Migrating Contact}

During the cycle of loading-unloading in walking or running, the contact area between the articular cartilage layers changes and moves (Iseki and Tomatsu, 1976). Therefore, the so-called problem of migrating contact can be formulated (Chen et al., 2009; Argatov, 2012b). In the framework of the asymptotic model [equations (3)-(6)], such a problem was considered and an approximate solution was given for the steady-state regime. However, the corresponding problem of migrating contact for a viscoelastic/biphasic layer bonded to a rigid sphere periodically moving with rotation over the surface of another viscoelastic/biphasic layer bonded to a rigid flat base has not been investigated, yet even in the small thickness approximation.

\subsection{Curved Layer Model}

In particular, for the hip joint, the case of spherical geometry of the articular cartilage layer is very important. The corresponding contact was studied in the thin layer approximation (Argatov, 2011). Also, an approximate analysis of the deformation problem for a hemispherical biphasic layer was attempted recently by Quinonez et al. (2011). Note that, in the case of a curved compressible elastic layer, the first-order asymptotic theory was developed by Mal'kov (1998). However, the generalization of the asymptotic model [equation (1)] for a thin biphasic layer bonded to a rigid base 
shaped as an elliptic paraboloid is absent, and, correspondingly, the generalization of the asymptotic model [equations (3)-(6)], which takes into account the effect of the cartilage layer curvature is still missing. On the other hand, there is one more interesting outlook in this specific topic. What happens when there are two contact areas, which may interact? Looking at MR images of the ankle joint (Li et al., 2008), it seems quite a common situation that two contact areas merge under in vivo loading conditions.

\subsection{Bone Deformation}

The asymptotic model [equations (3)-(6)] neglects the deformation of the subchondral bones. However, for the case of intensive loading of the joint, the deformation of the bones may contribute to the contact pressure pattern. This effect was not analytically studied yet, though in the FE simulations usually (Anderson et al., 2010; Duarte et al., 2015) the bones are assumed to be compliant with relatively large elastic modulus compared to the elastic modulus of the articular cartilage layers. Here, it should be noted that under the dynamic loading (Laasanen et al., 2003; Park et al., 2004), the so-called dynamic elastic modulus of cartilage is much higher than that measured under quasi-static conditions.

\subsection{Synovial Fluid Effect}

The asymptotic model [equations (3)-(6)] assumes direct contact between the two cartilage layers, which can occur after some time when the synovial fluid is squeezed out of the contact region (Ateshian et al., 1994). The problem of squeezing of the synovial fluid was studied in a number of papers (Hou et al., 1992; Ruggiero et al., 2011; Yousfi et al., 2013). Of considerable practical interest is a generalization of the asymptotic model [equations (3)-(6)] that accounts for the synovial fluid effect in non-axisymmetric configuration. One concern is about the exudation of the interstitial fluid out from under the cartilage layers contact area (Caligaris and Ateshian, 2008). How important is the effect of the migrating boundaries on the fluid pressurization, and what boundary conditions should be imposed with respect to the contact pressure distribution?

\subsection{Damage Accumulation and Impact-Induced Fissuring}

The deformation problem for a biphasic layer (Figure 1A) was considered under quasi-static loading (Ateshian et al., 1994) and though neglecting the inertia effect, it can be applied to study the contact between cartilage layers under dynamic loading ( $\mathrm{Wu}$ et al., 1998; Quinn et al., 2001) and impact loading (Jeffrey et al., 1995; Ewers et al., 2001) under normal physiological conditions (Aspden et al., 2002). As a first approximation, it was suggested (Argatov and Mishuris, 2015a) that the asymptotic model [equations (3)-(6)] can predict the deformation of articular cartilage and the damage accumulation process until the fracture moment. Of course, the further development of mathematical models for impact-induced fissuring (Atkinson et al., 1998; Kafka, 2002) will require a more sophisticated mathematical modeling framework [see, e.g., Peña (2011) and Mengoni and Ponthot (2015)]. Nevertheless, this simple model reveals the key model parameters, which should be reported in the experimental studies in order to facilitate the comparison between different experiments.

\subsection{Shear Loading}

The asymptotic model [equations (3)-(6)] considers the case of unilateral normal frictionless contact and is based on the asymptotic solution [equation (1)] of the deformation problem for a biphasic layer loaded by a normal distributed load. Due to a very small coefficient of friction for articular cartilage layers in contact via a film of synovial fluid, the tangential stresses are usually neglected in evaluation of the stress-strain state of the joint in physiologically normal conditions (Ateshian, 2009). However, under severe loading, e.g., in traumatic situations in sport, the cartilage layers can transform a significant shear loading (Carter and Wong, 1988). Therefore, the problem of tangential loading of a thin biphasic layer requires a special attention.

\subsection{Non-Homogeneity}

As it is known (Poole et al., 2001), articular cartilage is a nonhomogeneous tissue with properties primarily varying with depth (Schinagl et al., 1997). Recently, the problem of normal loading of a thin biphasic layer was studied by (Vitucci et al., 2016) for a special case of exponential type of non-homogeneity. The obtained asymptotic solution can be used for generalizing the asymptotic model of uniateral contact [equations (3)-(6)] for this case (Vitucci and Mishuris, 2016).

\subsection{Whole Joint Analytical Model}

It is a very difficult problem to create a system of analytical models (let us say, sub-models), which account for the major mechanical aspects of articular contact in the knee joint, e.g., including the deformation of patella, menisci, and ligaments (Maquet, 1976). The aim of such asymptotic mathematical modeling is to predict (at least by the order of magnitude) the contact forces and the deformation factors in the joint for a range of physiological displacements of the bones.

\subsection{Fibril-Reinforced Material Models}

By accounting for the microstructure of articular cartilage, in the literature, a number of advanced material models for describing the deformation of cartilage have been presented (Korhonen et al., 2003; Wilson et al., 2005b; Freutel et al., 2014). Since the use of these models for analytical solution is confronted with considerable mathematical difficulties, it makes sense to solve the model deformation problem (Figure 1A) for a relatively thin fibril-reinforced layer by FE methods and highlighting the differences with the basic homogeneous case first studied by Armstrong (1986) and Ateshian et al. (1994) using an analytical technique.

\subsection{FEM-Based Surrogate Models}

It goes without saying that the articular cartilage contact problem with realistic geometry and that takes into account the effects (discussed above) represents a challenge for a real-time computer simulations of the knee joint dynamics in real activities such as the gait cycle (Pérez-González et al., 2008). In special cases, e.g., under the assumption of cyclic dynamic loading during walking when the contact loading configuration is repeated, the result of certain blocks of the computational algorithm can be fitted with computationally cheap surrogate contact analytical models. 
Such an approach, introduced by Lin et al. (2010), is called surrogate modeling. It is foreseen that the asymptotic models can be used for developing surrogate models for impact loading.

\subsection{Contact of Articular Cartilage with Implants}

The asymptotic model [equations (3)-(6)] covers the case of unilateral frictionless contact between the cartilage layers, and it was applied to study the difference in the contact pressure patterns in the normal and pathological (early stage of osteoarthritis) situations (Wu et al., 2000). In a marginal pathological situation, a part of the diseased cartilage can be replaced by an artificial tissue (Hung et al., 2003; Mano and Reis, 2007) or even with a metallic implant (Manda and Eriksson, 2012). The corresponding contact problems did not receive much attention so far (Hale et al., 1993; Owen and Wayne, 2011; Manda and Eriksson, 2014).

\subsection{Multiscale Structural Modeling of Articular Cartilage}

The asymptotic model [equations (3)-(6)] can be regarded as a "rude" model, since it operates with average constant parameters and does not reflect the actual microstructure of articular cartilage, which represents an important factor in the pathogenesis of osteoarthritis (Buckwalter et al., 2013). From the point of view of multiscale modeling, this asymptotic model represents a macro-level, and to our knowledge, no link has been established with micro-level modeling framework. A certain progress in this direction was made by Federico et al. (2005) who constructed the TITH model by employing the homogenization scheme developed for fiber-reinforced elastic composite materials. The next step can be made by utilizing the recent theoretical development for poroelastic materials (Hellmich et al., 2009; Ortega et al., 2010).

\section{CONCLUSION}

The asymptotic models presented and discussed above are generally nowadays regarded as oversimplified mathematical models.

\section{REFERENCES}

Anderson, A. E., Ellis, B. J., Maas, S. A., and Weiss, J. A. (2010). Effects of idealized joint geometry on finite element predictions of cartilage contact stresses in the hip. J. Biomech. 43, 1351-1357. doi:10.1016/j.jbiomech.2010. 01.010

Argatov, I. (2011). A general solution of the axisymmetric contact problem for biphasic cartilage layers. Mech. Res. Comm. 38, 29-33. doi:10.1016/j. mechrescom.2010.11.006

Argatov, I. (2013a). Contact problem for a thin elastic layer with variable thickness: application to sensitivity analysis of articular contact mechanics. Appl. Math. Model. 37, 8383-8393. doi:10.1016/j.apm.2013.03.042

Argatov, I., and Mishuris, G. (2010). Axisymmetric contact problem for a biphasic cartilage layer with allowance for tangential displacements on the contact surface. Eur. J. Mech. A Solids 29, 1051-1064. doi:10.1016/j.euromechsol.2010. 07.003

Argatov, I., and Mishuris, G. (2011a). Elliptical contact of thin biphasic cartilage layers: exact solution for monotonic loading. J. Biomech. 44, 759-761. doi:10.1016/j.jbiomech.2010.11.010

Argatov, I., and Mishuris, G. (2011b). Contact problem for thin biphasic cartilage layers: perturbation solution. Quart. J. Mech. Appl. Math. 64, 297-318. doi:10.1093/qjmam/hbr008
By all means, the articular contact mechanics should not be confronted by asymptotic modeling alone. At the same time, asymptotic models can be successfully used to facilitate FEM analysis. It is to emphasize that each asymptotic model yields an approximate solution to a problem under consideration, whose accuracy and robustness can be evaluated when the exact solution (analytical or numerical) is available [see, e.g., the examples of refined contact problem and its asymptotic model studied by Mishuris and Argatov (2009)]. One benefit of using simplified (with a limited number of parameters) models, which by construction preserve essential features of the contact system captured by more complex mathematical models, is that they can be employed for uncertainty quantification, when detailed mechanical and geometrical aspects of the system are not fully known. Simple as they are, such models provide a robust theoretical framework for the preliminary analysis of the experimental data as well as for controlling computer simulations produced on the basis of more complicated mathematical models.

\section{AUTHOR CONTRIBUTIONS}

IA and GM worked on each part of this paper together.

\section{ACKNOWLEDGMENTS}

The authors are grateful to Gennaro Vitucci for critically reading the manuscript and helpful discussion.

\section{FUNDING}

IA is grateful to the DAAD (German Academic Exchange Service - Deutscher Akademischer Austausch Dienst) for financial support during his stay at the TU Berlin. GM acknowledges the EU project HORIZON2020 RISE Marie Sklodowska Curie grant MATRIXASSAY No. 644175 for partial financial support during IA's visit to Aberystwyth University.

Argatov, I., and Mishuris, G. (2011c). Frictionless elliptical contact of thin vis coelastic layers bonded to rigid substrates. Appl. Math. Model. 35, 3201-3212. doi:10.1016/j.apm.2011.01.029

Argatov, I., and Mishuris, G. (2015a). A phenomenological model of damage in articular cartilage under impact loading. Mech. Res. Comm. 69, 87-90. doi:10.1016/j.mechrescom.2015.06.013

Argatov, I., and Mishuris, G. (2015b). Contact Mechanics of Articular Cartilage Layers. Cham: Springer.

Argatov, I., and Mishuris, G. (2015d). "Impact-induced fissuring of articular cartilage: an asymptotic modelling study," in The 3rd Polish Congress of Mechanics, September 8th-11th, 2015 (Gdañsk, Poland).

Argatov, I., and Mishuris, G. (2016). An asymptotic model for a thin bonded elastic layer coated with an elastic membrane. Appl. Math. Model. 40, 2541-2548. doi:10.1016/j.apm.2015.09.109

Argatov, I. I. (2012a). Development of an asymptotic modeling methodology for tibio-femoral contact in multibody dynamic simulations of the human knee joint. Multibody Syst. Dyn. 28, 3-20. doi:10.1007/s11044-011-9275-6

Argatov, I. I. (2012b). "An analytical model for frictionless contact between thin transversely isotropic viscoelastic layers with a migrating contact area: application to repetitive movements of the knee joint during walking," in The 2nd Joint International Conference on Multibody System Dynamics, May 29 - June 1, 2012 (Stuttgart, Germany). 
Argatov, I. I. (2013b). Mathematical modeling of linear viscoelastic impact: application to drop impact testing of articular cartilage. Trib. Int. 63, 213-225. doi:10.1016/j.triboint.2012.09.015

Argatov, I. I., and Mishuris, G. S. (2015c). An asymptotic model for a thin biphasic poroviscoelastic layer. Quart. J. Mech. Appl. Math. 68, 289-297. doi:10.1093/ qjmam/hbv008

Argatov, I. I., Mishuris, G. S., and Popov, V. L. (2016a). Asymptotic modeling of the JKR adhesion contact for a thin elastic layer. Quart. J. Mech. Appl. Math.69, 161-179. doi:10.1093/qjmam/hbw002

Argatov, I. I., Selyutina, N. S., and Mishuris, G. S. (2016b). Impact problem for the quasi-linear viscoelastic standard solid model. J. Strain Anal. Eng. Des. 51, 294-303. doi:10.1177/0309324715610027

Armstrong, C. (1986). An analysis of the stresses in a thin layer of articular cartilage in a synovial joint. Eng. Med. 15, 55-61. doi:10.1243/EMED_JOUR_1986_015_ 018_02

Aspden, R. M., Jeffrey, J. E., and Burgin, L. V. (2002). Impact loading: physiological or pathological? Osteoarthr. Cartilage 10, 588-589. doi:10.1053/joca.2002.0803

Ateshian, G. A. (2009). The role of interstitial fluid pressurization in articular cartilage lubrication. J. Biomech. 42, 1163-1176. doi:10.1016/j.jbiomech.2009. 04.040

Ateshian, G. A., Lai, W. M., Zhu, W. B., and Mow, V. C. (1994). An asymptotic solution for the contact of two biphasic cartilage layers. J. Biomech. 27, 1347-1360. doi:10.1016/0021-9290(94)90044-2

Atkinson, T. S., Haut, R. C., and Altiero, N. J. (1998). Impact-induced fissuring of articular cartilage: an investigation of failure criteria. J. Biomech. Eng. 120, 181-187. doi:10.1115/1.2798025

Barry, S. I., and Holmes, M. (2001). Asymptotic behaviour of thin poroelastic layers. IMA J. Appl. Math. 66, 175-194. doi:10.1093/imamat/66.2.175

Bei, Y., and Fregly, B. J. (2004). Multibody dynamic simulation of knee contact mechanics. Med. Eng. Phys. 26, 777-789. doi:10.1016/j.medengphy.2004.07.004

Blankevoort, L., Kuiper, J. H., Huiskes, R., and Grootenboer, H. J. (1991). Articular contact in a three-dimensional model of the knee. J. Biomech. 24, 1019-1031. doi:10.1016/0021-9290(91)90019-J

Buckwalter, J. A., Anderson, D. D., Brown, T. D., Tochigi, Y., and Martin, J. A. (2013). The roles of mechanical stresses in the pathogenesis of osteoarthritis: implications for treatment of joint injuries. Cartilage 4, 286-294. doi:10.1177/ 1947603513495889

Bursać, P., McGrath, C. V., Eisenberg, S. R., and Stamenović, D. (2000). A microstructural model of elastostatic properties of articular cartilage in confined compression. J. Biomech. Eng. 122, 347-353. doi:10.1115/1.1286561

Caligaris, M., and Ateshian, G. A. (2008). Effects of sustained interstitial fluid pressurization under migrating contact area, and boundary lubrication by synovial fluid, on cartilage friction. Osteoarthr. Cartilage 16, 1220-1227. doi:10.1016/j. joca.2008.02.020

Carter, D. R., Beaupre, G. S., Wong, M., Smith, R. L., Andriacchi, T. P., and Schurman, D. L. (2004). The mechanobiology of articular cartilage development and degeneration. Clin. Orthop. Relat. Res. 427S, S69-S77. doi:10.1097/01.blo. 0000144970.05107.7e

Carter, D. R., and Wong, M. (1988). The role of mechanical loading histories in the development of diarthrodial joints. J. Orthop. Res. 6, 804-816. doi:10.1002/jor. 1100060604

Caruntu, D. I., and Hefzy, M. S. (2004). 3-D anatomically based dynamic modeling of the human knee to include tibio-femoral and patello-femoral joints. J. Biomech. Eng. 126, 44-53. doi:10.1115/1.1644565

Chen, X., Sunagawa, K., and Hisada, T. (2009). Development of a finite element contact analysis algorithm for charged-hydrated soft tissues with large sliding. Int. J. Numer. Methods Eng. 78, 483-504. doi:10.1002/nme.2497

Curnier, A., He, Q.-C., and Zysset, P. (1995). Conewise linear elastic materials. J. Elast. 37, 1-38. doi:10.1007/BF00043417

Delp, S. L., and Loan, J. P. (2000). A computational framework for simulating and analyzing human and animal movement. IEEE Comput. Sci. Eng. 2, 46-55. doi:10.1109/5992.877394

Duarte, R. J., Ramos, A., Completo, A., Relvas, C., and Simões, J. A. (2015). The importance of femur/acetabulum cartilage in the biomechanics of the intact hip: experimental and numerical assessment. Comput. Methods Biomech. Biomed. Eng. 18, 880-889. doi:10.1080/10255842.2013.854335

Eberhard, P., Spägele, T., and Gollhofer, A. (1999). Investigations for the dynamical analysis of human motion. Multibody Syst. Dyn. 3, 1-20. doi:10.1023/A: 1009880222265
Eberhardt, A. W., Keer, L. M., Lewis, J. L., and Vithoontien, V. (1990). An analytical model of joint contact. J. Biomech. Eng. 112, 407-413. doi:10.1115/1.2891204

Ewers, B. J., Dvoracek-Driksna, D., Orth, M. W., and Haut, R. C. (2001). The extent of matrix damage and chondrocyte death in mechanically traumatized articular cartilage explants depends on rate of loading. J. Orthop. Res. 19, 779-784. doi:10.1016/S0736-0266(01)00006-7

Fahmy, N. R., Williams, E. A., and Noble, J. (1983). Meniscal pathology and osteoarthritis of the knee. J. Bone Joint Surg. Br. 65-B, 24-28.

Federico, S., Grillo, A., La Rosa, G., Giaquinta, G., and Herzog, W. (2005). A transversely isotropic, transversely homogeneous microstructural-statistical model of articular cartilage. J. Biomech. 38, 2008-2018. doi:10.1016/j.jbiomech.2004. 09.020

Flores, P., Machado, M., Silva, M. T., and Martins, J. M. (2011). On the continuous contact force models for soft materials in multibody dynamics. Multibody Syst. Dyn. 25, 357-375. doi:10.1007/s11044-010-9237-4

Freutel, M., Schmidt, H., Dürselen, L., Ignatius, A., and Galbusera, F. (2014). Finite element modeling of soft tissues: material models, tissue interaction and challenges. Clin. Biomech. (Bristol, Avon) 29, 363-372. doi:10.1016/j.clinbiomech 2014.01.006

Galbusera, F., Bashkuev, M., Wilke, H.-J., Shirazi-Adl, A., and Schmidt, H. (2014) Comparison of various contact algorithms for poroelastic tissues. Comput Methods Biomech. Biomed. Eng. 17, 1323-1334. doi:10.1080/10255842.2012. 745858

Garcia, J., Altiero, N., and Haut, R. (2008). An approach for the stress analysis of transversely isotropic biphasic cartilage under impact load. J. Biomech. Eng. 120, 608-613. doi:10.1115/1.2834751

Guess, T. M., Thiagarajan, G., Kia, M., and Mishra, M. (2010). A subject specific multibody model of the knee with menisci. Med. Eng. Phys. 32, 505-515. doi:10.1016/j.medengphy.2010.02.020

Hale, J. E., Rudert, M. J., and Brown, T. D. (1993). Indentation assessment of biphasic mechanical property deficits in size-dependent osteochondral defect repair. J. Biomech. 26, 1319-1325. doi:10.1016/0021-9290(93)90355-I

Hellmich, C., Celundova, D., and Ulm, F. (2009). Multiporoelasticity of hierarchically structured materials: micromechanical foundations and application to bone. J. Eng. Mech. 135, 382-394. doi:10.1061/(ASCE)EM.1943-7889.0000001

Herzog, W., and Federico, S. (2006). Considerations on joint and articular cartilage mechanics. Biomech. Model. Mechanobiol. 5, 64-81. doi:10.1007/s10237-0060029-y

Hlaváček, M. (1999). A note on an asymptotic solution for the contact of two biphasic cartilage layers in a loaded synovial joint at rest. J. Biomech. 32, 987-991. doi:10.1016/S0021-9290(99)00082-2

Hou, J. S., Mow, V. C., Lai, W. M., and Holmes, M. H. (1992). An analysis of the squeeze-film lubrication mechanism for articular cartilage. J. Biomech. 25, 247-259. doi:10.1016/0021-9290(92)90024-U

Hung, C. T., Lima, E. G., Mauck, R. L., Taki, E., LeRoux, M. A., Lu, H. H., et al. (2003). Anatomically shaped osteochondral constructs for articular cartilage repair. J. Biomech. 36, 1853-1864. doi:10.1016/S0021-9290(03)00213-6

Hunt, K. H., and Crossley, F. R. E. (1975). Coefficient of restitution interpreted as damping in vibroimpact. ASME J. Appl. Mech. 42, 440-445. doi:10.1115/1. 3423596

Iseki, F., and Tomatsu, T. (1976). The biomechanics of the knee joint with special reference to the contact area. Keio. J. Med. 25, 37-44. doi:10.2302/kjm.25.37

Jeffrey, J. E., Gregory, D. W., and Aspden, R. M. (1995). Matrix damage and chondrocyte viability following a single impact load on articular cartilage. Arch. Biochem. Biophys. 322, 87-96. doi:10.1006/abbi.1995.1439

Johnson, K. L. (1985). Contact Mechanics. Cambridge, UK: Cambridge University Press.

Kafka, V. (2002). Surface fissures in articular cartilage: new concepts, hypotheses and modeling. Clin. Biomech. 17, 73-80. doi:10.1016/S0268-0033(01)00095-X

Kessler, M. A., Glaser, C., Tittel, S., Reiser, M., and Imhoff, A. B. (2006). Volume changes in the menisci and articular cartilage of runners: an in vivo investigation based on 3-D magnetic resonance imaging. Am. J. Sports Med. 34, 832-836. doi: $10.1177 / 0363546505282622$

Koo, S., and Andriacchi, T. P. (2007). A comparison of the influence of global functional loads vs. local contact anatomy on articular cartilage thickness at the knee. J. Biomech. 40, 2961-2966. doi:10.1016/j.jbiomech.2007.02.005

Korhonen, R. K., Laasanen, M. S., Töyräs, J., Lappalainen, R., Helminen, H. J., and Jurvelin, J. S. (2003). Fibril reinforced poroelastic model predicts specifically mechanical behavior of normal, proteoglycan depleted and collagen 
degraded articular cartilage. J. Biomech. 36, 1373-1379. doi:10.1016/S00219290(03)00069-1

Korhonen, R. K., Wong, M., Arokoski, J., Lindgren, R., Helminen, H. J., Hunziker, E. B., et al. (2002). Importance of the superficial tissue layer for the indentation stiffness of articular cartilage. Med. Eng. Phys. 24, 99-108. doi:10.1016/S13504533(01)00123-0

Laasanen, M. S., Töyräs, J., Korhonen, R. K., Rieppo, J., Saarakkala, S., Nieminen, M. T., et al. (2003). Biomechanical properties of knee articular cartilage. Biorheology $40,133-140$.

Lai, W. M., Hou, J. S., and Mow, V. C. (1991). A triphasic theory for the swelling and deformation behaviors of articular cartilage. J. Biomech. Eng. 113, 245-258. doi:10.1115/1.2894880

Li, G., Wan, L., and Kozanek, M. (2008). Determination of real-time in-vivo cartilage contact deformation in the ankle joint. J. Biomech. 41, 128-136. doi:10.1016/j.jbiomech.2007.07.006

Lin, Y.-Ch, Haftka, R. T., Queipo, N. V., and Fregly, B. J. (2010). Surrogate articular contact models for computationally efficient multibody dynamic simulations. Med. Eng. Phys. 32, 584-594. doi:10.1016/j.medengphy.2010.02. 008

Machado, M., Flores, P., Ambrosio, J., and Completo, A. (2011). Influence of the contact model on the dynamic response of the human knee joint. Proc. Inst. Mech. Eng. KJ Multibody Dyn. 225, 344-358.

Machado, M., Flores, P., Claro, J. C. P., Ambrósio, J., Silva, M., Completo, A., et al. (2010). Development of a planar multibody model of the human knee joint. Nonlinear Dyn. 60, 459-478. doi:10.1007/s11071-009-9608-7

Mal'kov, V. M. (1998). Mechanics of Multi-Layer Elastomeric Constructions. St. Petersburg: St. Petersburg University Publication. [in Russian].

Maly, M. R., Costigan, P. A., and Olney, S. J. (2008). Mechanical factors relate to pain in knee osteoarthritis. Clin. Biomech. 23, 796-805. doi:10.1016/j.clinbiomech. 2008.01.014

Manda, K., and Eriksson, A. (2012). Time-dependent behavior of cartilage surrounding a metal implant for full-thickness cartilage defects of various sizes: a finite element study. Biomech. Model. Mechanobiol. 11, 731-742. doi:10.1007/ s10237-011-0346-7

Manda, K., and Eriksson, A. (2014). Modeling of constrained articular cartilage growth in an intact knee with focal knee resurfacing metal implant. Biomech. Model. Mechanobiol. 13, 599-613. doi:10.1007/s10237-013-0521-0

Mano, J. F., and Reis, R. L. (2007). Osteochondral defects: present situation and tissue engineering approaches. J. Tissue Eng. Regen. Med. 1, 261-273. doi:10.1002/term.37

Maquet, P. G. J. (1976). Biomechanics of the Knee. Berlin, Heidelberg, New York: Springer-Verlag.

Mengoni, M., and Ponthot, J. P. (2015). A generic anisotropic continuum damage model integration scheme adaptable to both ductile damage and biological damage-like situations. Int. J. Plast. 66, 46-70. doi:10.1016/j.ijplas.2014. 04.005

Mishuris, G., and Argatov, I. (2009). "Exact solution to a refined contact problem for biphasic cartilage layers," in Proc. 1st International Conference "Mathematical and Computational Biomedical Engineering" - CMBE2009 June 29-July 1, 2009, eds P. Nithiarasu and R. Löhner (Swansea, UK), 151-154.

Monteiro, N. M. B., da Silva, M. P. T., Folgado, J. O. M. G., and Melancia, J. P. L. (2011). Structural analysis of the intervertebral discs adjacent to an interbody fusion using multibody dynamics and finite element cosimulation. Multibody Syst. Dyn. 25, 245-270. doi:10.1007/s11044-010-9226-7

Morel, V., and Quinn, T. M. (2004). Cartilage injury by ramp compression near the gel diffusion rate. J. Orthop. Res. 22, 145-151. doi:10.1016/S0736-0266(03) 00164-5

Mow, V. C., and Guo, X. E. (2002). Mechano-electrochemical properties of articular cartilage: their inhomogeneities and anisotropies. Annu. Rev. Biomed. Eng. 4, 175-209. doi:10.1146/annurev.bioeng.4.110701.120309

Mow, V. C., Kuei, S. C., Lai, W. M., and Armstrong, C. G. (1980). Biphasic creep and stress relaxation of articular cartilage in compression: theory and experiments. J. Biomech. Eng. 102, 73-84. doi:10.1115/1.3138202

Ortega, J. A., Ulm, F.-J., and Abousleiman, Y. (2010). The effect of particle shape and grain-scale properties of shale: A micromechanics approach. Int. J. Numer. Anal. Methods Geomech. 34, 1124-1156. doi:10.1002/nag.850

Owen, J. R., and Wayne, J. S. (2011). Contact models of repaired articular surfaces: influence of loading conditions and the superficial tangential zone. Biomech. Model. Mechanobiol. 10, 461-471. doi:10.1007/s10237-010-0247-1
Park, S., Hung, C. T., and Ateshian, G. A. (2004). Mechanical response of bovine articular cartilage under dynamic unconfined compression loading at physiological stress levels. Osteoarthr. Cartilage 12, 65-73. doi:10.1016/j.joca.2003.08.005

Peña, E. (2011). Damage functions of the internal variables for soft biological fibred tissues. Mech. Res. Commun. 38, 610-615. doi:10.1016/j.mechrescom. 2011.09.002

Peña, E., Calvo, B., Martínez, M. A., and Doblaré, M. (2006). A three-dimensional finite element analysis of the combined behavior of ligaments and menisci in the healthy humen knee joint. J. Biomech. 39, 1686-1701. doi:10.1016/j.jbiomech. 2005.04.030

Pérez-González, A., Fenollosa-Esteve, C., Sancho-Bru, J. L., Sánchez-Marín, F. T., Vergara, M., and Rodríguez-Cervantes, P. J. (2008). A modified elastic foundation contact model for application in 3D models of the prosthetic knee. Med. Eng. Phys. 30, 387-398. doi:10.1016/j.medengphy.2007.04.001

Poole, A. R., Kojima, T., Yasuda, T., Mwale, F., Kobayashi, M., and Laverty, S. (2001). Composition and structure of articular cartilage: a template for tissue repair (review). Clin. Orthop. Relat. Res. 391(Suppl.), S26-S33. doi:10.1097/00003086200110001-00004

Quinn, T. M., Allen, R. G., Schalet, B. J., Perumbuli, P., and Hunziker, E. B. (2001). Matrix and cell injury due to sub-impact loading of adult bovine articular cartilage explants: effects of strain rate and peak stress. J. Orthop. Res. 19, 242-249. doi:10.1016/S0736-0266(00)00025-5

Quinonez, A., Summers, J., Fisher, J., and Jin, Z. (2011). An analytical solution for the radial and tangential displacements on a thin hemispherical layer of articular cartilage. Biomech. Model. Mechanobiol. 10, 283-293. doi:10.1007/s10237-0100234-6

Rogosin, S., Mishuris, G., Koroleva, A., and Vinakurava, A. (2016). Analysis of the unilateral contact problem for biphasic cartilage layers with an elliptic contact zone and accounting for tangential displacements. Math. Modell. Anal. 21, 585-609. doi:10.3846/13926292.2016.1196249

Rosneck, J., Higuera, C. A., Tadross, N., Krebs, V., and Barsoum, W. K. (2007). Managing knee osteoarthritis before and after arthroplasty. Cleve. Clin. J. Med. 74, 663-671.

Ruggiero, A., Gómez, E., and D’Amato, R. (2011). Approximate analytical model for the squeeze-film lubrication of the human ankle joint with synovial fluid filtrated by articular cartilage. Trib. Lett. 41, 337-343. doi:10.1007/s11249-010-9710-5

Schinagl, R. M., Gurskis, D., Chen, A. C., and Sah, R. L. (1997). Depth-dependent confined compression modulus of full-thickness bovine articular cartilage. J. Orthop. Res. 15, 499-506. doi:10.1002/jor.1100150404

Selyutina, N. S., Argatov, I. I., and Mishuris, G. S. (2015). On application of Fung's quasi-linear viscoelastic model to modeling of impact experiment for articular cartilage. Mech. Res. Commun. 67, 24-30. doi:10.1016/j.mechrescom. 2015.04.003

Silva, M. P. T., Ambrósio, J. A. C., and Pereira, M. S. (1997). Biomechanical model with joint resistance for impact simulation. Multibody Syst. Dyn. 1, 65-84. doi:10.1023/A:1009700405340

Soltz, M. A., and Ateshian, G. A. (2000). A conewise linear elasticity mixture model for the analysis of tension-compression nonlinearity in articular cartilage. J. Biomech. Eng. 122, 576-586. doi:10.1115/1.1324669

van den Bogert, A. J., Read, L., and Nigg, B. M. (1999). An analysis of hip joint loading during walking, running, and skiing. Med. Sci. Sports Exerc. 31, 131-142. doi:10.1097/00005768-199901000-00021

Varga, F., Drzik, M., Handl, M., Chlpik, J., Kos, P., Filova, E., et al. (2007). Biomechanical characterization of cartilages by a novel approach of blunt impact testing. Physiol. Res. 56(Suppl. 1), S61-S68.

Vitucci, G., Argatov, I., and Mishuris, G. (2016). An asymptotic model for the deformation of a transversely isotropic, transversely homogeneous biphasic cartilage layer. Math. Methods Appl. Sci. doi:10.1002/mma.3895

Vitucci, G., and Mishuris, G. (2016). "3-D contact problem of a transversely isotropic, transversely homogeneous biphasic cartilage layer," in International Workshop "Multi-Scale and Multi-Physics Testing of High-Performance Materials" (Germany: Technische Universität Berlin). February 18-19, 2016.

Wilson, W., van Donkelaar, C. C., van Rietberger, R., and Huiskes, R. (2005a). The role of computational models in the search for the mechanical behaviour and damage mechanisms of articular cartilage. Med. Eng. Phys. 27, 810-826. doi:10.1016/j.medengphy.2005.03.004

Wilson, W., van Donkelaar, C. C., van Rietberger, R., and Huiskes, R. (2005b). A fibril-reinforced poroviscoelastic swelling model for articular cartilage. J. Biomech. 38, 1195-1204. doi:10.1016/j.jbiomech.2004.07.003 
Wu, J. Z., and Herzog, W. (2000). On the pressure gradient boundary condition for the contact of two biphasic cartilage layers. J. Biomech. 33, 1331-1332. doi:10.1016/S0021-9290(99)00224-9

Wu, J. Z., Herzog, W., and Epstein, M. (1997). Evaluation of the finite element software ABAQUS for biomechanical modelling of biphasic tissues. J. Biomech. 31, 165-169. doi:10.1016/S0021-9290(97)00117-6

Wu, J. Z., Herzog, W., and Epstein, M. (1998). Articular joint mechanics with biphasic cartilage layers under dynamic loading. J. Biomech. Eng. 120, 77-84. doi:10.1115/1.2834310

Wu, J. Z., Herzog, W., and Epstein, M. (2000). Joint contact mechanics in the early stages of osteoarthitis. Med. Eng. Phys. 22, 1-12. doi:10.1016/S1350-4533(00) 00012-6

Wu, J. Z., Herzog, W., and Ronsky, J. (1996). Modeling axi-symmetrical joint contact with biphasic cartilage layers-An asymptotic solution. J. Biomech. 29, 1263-1281. doi:10.1016/0021-9290(96)00051-6
Yousfi, M., Bou-Said, B., and Tichy, J. (2013). An analytical study of the squeezing flow of synovial fluid. Mech. Ind. 14, 59-69. doi:10.1051/meca/ 2012044

Conflict of Interest Statement: The authors declare that the research was conducted in the absence of any commercial or financial relationships that could be construed as a potential conflict of interest.

Copyright (๑) 2016 Argatov and Mishuris. This is an open-access article distributed under the terms of the Creative Commons Attribution License (CC BY). The use, distribution or reproduction in other forums is permitted, provided the original author(s) or licensor are credited and that the original publication in this journal is cited, in accordance with accepted academic practice. No use, distribution or reproduction is permitted which does not comply with these terms. 